\title{
A hardware multiplier/divider for the PDP 8S computer
}

\author{
P. A. V. THOMAS and N. BALATONI* \\ Electrical Engineering Department, University of Windsor \\ Windsor, Ontario, Canada
}

This paper outlines the design of a multiplier/divider being developed for the PDP-8/S. The unit will carry out the required function on two 12 -bit signed numbers (using 2's complements for negative numbers) and produce a correctly signed result. All data transfers will be through the programmed I/O channel, no data break facility being required. The estimated time taken for the operations is 96 microsec for multiplication and 176 microsec for division, which is considered reasonable in comparison to the $\mathrm{I} / \mathrm{O}$ times of $38 \mathrm{microsec}$. The total material cost is estimated at $\$ 1,500$. Some variations of the basic unit are also discussed.

\section{INTRODUCTION}

For some time now it has been considered desirable to have a hardware multiplier for the PDP:8/S rather than having to rely on the use of the software package, this being particularly true when operating in a real-time on-line mode. Although there exists the extended arithmetic element, this is not available to the user of the PDP-8/S due to its requirement of the data break facility, which is not part of the standard equipment. Therefore, it was decided to develop a suitable unit using the programmed I/O channel, if this could be achieved economically and with a reasonable overall speed of operation.

As the design progressed, it became apparent that, at relatively little increase in cost, a divider could also be included even though its operation would be slower than that of the multiplier, which was the main concern.

\section{GENERAL ARRANGEMENT}

It was decided that the unit should permit the multiplication or division of two 12-bit signed numbers, using 2 's complement form for negative numbers, and produce a correctly signed result.

Due to the low speed of the I/O channel (38 microsec/operation), it would not be practical to use the computer accumulator as part of the unit; instead it was necessary to provide three 12-bit shifting registers to hold the initial data and generated results. All data transfers to and from these registers were conveniently carried out by standard programmed I/O commands. It was also necessary to provide an adder/subtractor unit to carry out the required arithmetic and

* The authors acknowledge the financial support given to this project by the National Research Council of Canada. counters and logic to carry out the calculation algorithms.

The whole design was essentially based on the multiplication operation-the primary purpose of the unit-and the method used is now developed.

\section{MULTIPLICATION}

Basically, multiplication may be carried out in parallel or serial mode, and, although the former is much faster, it is also much more costly and was, therefore, rejected. In the case of a serial multiplier, the normal mode of operation is essentially one of continued addition, this being a relatively simple operation to perform. Unfortunately, in this simple scheme, only positive numbers may be used, negative numbers being handled by corrections. An alternative method is to use an algorithm developed by Booth and Booth (1965), which gives a correctly signed product of two signed numbers. Briefly, the method significant digits of the multiplier and either adding or subtracting the multiplicand to (or from) the partial product in the accumulator or leaving it unchanged. The contents of the multiplier register and accumulator are then shifted one place right, the above process being repeated 12 times for a 12-bit number. The complication of consists of examining the two least

this method is the necessity of requiring a subtractor in addition to an adder. The total operation time is thus essentially that required for the arithmetic operations, which may themselves be carried out in serial or parallel mode. The comparable overall times, with a clock rate of $1 \mathrm{MHz}$, will be approximately 200 or 72 microsec, respectively, but, again, the cost of the latter is much higher than that of the former. An alternative method, which is the one to be used, is an extension of Booth's method, developed by Thomas and Leech in 1959 and described in a PhD thesis (1961), which reduces the total time to about 96 microsec with serial arithmetic. This was considered reasonable in comparison to the other operation times.

In this method, the two least significant digits of the multiplier and a borrowed bit (initially set to zero) are compared, and the action carried out is determined according to the rules given in Table 1 , in which it is understood that the arithmetic operation is always between the partial product and the multiplicand. It will be observed from Table 1 that the $b$ digit of the $n$th operation is identical to the $m$ digit of the $(n-1)$ th operation, which means that the $b$ bit can be obtained as an extension of the multiplier shift register, as in this configuration the $m$ bit will automatically be shifted into the b-bit position after the multiplier register is shifted right two places, as required at the end of each cycle. Furthermore, the accumulator is also shifted right two places each cycle (except during the last, which has only a single shift), although one of the shifts may take place prior to the arithmetic operation, as indicated in Table 1. Thus, in each cycle we require an optional preshift and postshift pulse, in addition to a final shift pulse (excepting the last cycle).

It is because of this double shift that the total operation time of this method is halved.

The general arrangement of the registers and adder/subtractor is shown in Fig. 1, and the logic diagram of the Table 1

Multiplication Rules

\begin{tabular}{|c|c|c|c|c|}
\hline \multicolumn{3}{|c|}{ Multiplier Digits } & \multirow[b]{2}{*}{ Arithmetic Operation } & \\
\hline $\mathrm{m}$ & $\ell$ & b & & \\
\hline 0 & 0 & 0 & Do nothing & \\
\hline 0 & 1 & 0 & Addition & \\
\hline 1 & 0 & 0 & Subtraction after one shift & S Set \\
\hline 1 & 1 & 0 & Subtraction & $r_{b}=1$ \\
\hline 0 & 0 & 1 & Addition & Set \\
\hline 0 & 1 & 1 & Addition after one shift & $\{b=0$ \\
\hline 1 & 0 & 1 & Subtraction & \\
\hline 1 & 1 & 1 & Do nothing & \\
\hline
\end{tabular}

Note- $m$ and $\ell$ are the most and least significant digits of the least significant pair of the multiplier digits. $b$ is a borrowed bit. 


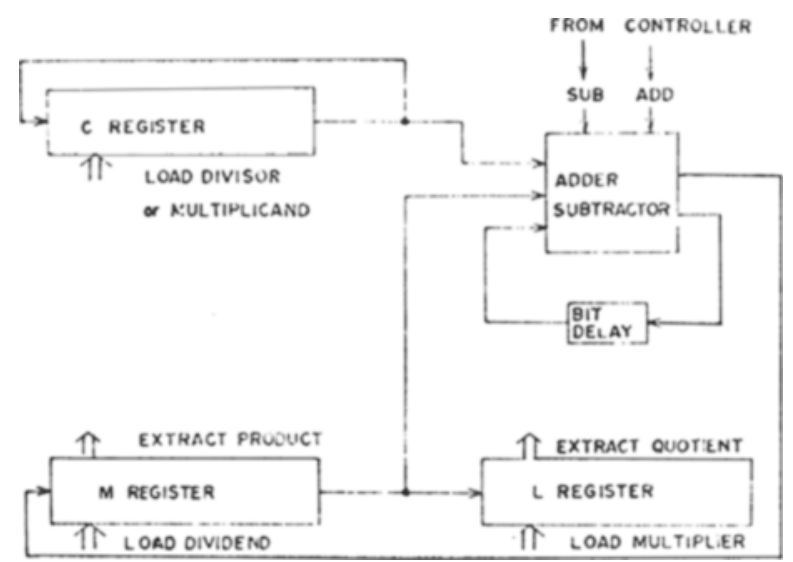

Fig. 1. General arrangement of registers and adder/subtractor.

algorithm controller in Fig. 2. The operation of the system will now be described.

The unit consists of three 12-bit shifting registers, $M$ and $L$ being the most and least significant halves of a double length accumulator and $\mathrm{C}$ being a "constant" recirculating register. Initially the multiplicand is loaded into the $\mathrm{C}$ register, the multiplier into the $\mathrm{L}$ register, and the $M$ register is cleared. At the same time as the multiplier is loaded, a pulse counter, cycle counter, and $\mathrm{L}_{\mathrm{b}}$ are cleared, and shortly afterwards a multiplier busy (MB) flip-flop is set, permitting the clock pulses to be gated into the pulse counter. This counter produces a sequence of pulses (A, B, $S_{1} \cdot S_{12}, X$, and $Y$ ) which are used to control an entire cycle. Typically, a cycle is as follows: Pulse A advances the cycle counter by unity, sets an add/subtract (AS) flip-flop to the add mode and a shift memory (SM) flip-flop to the postshift mode. Then Pulse B tests the three bits, $L_{m}, L_{\ell}$, and $L_{b}$, to determine the arithmetic operations (if any) and if a preshift is to be performed. If a subtraction is to be performed, the AS flip-flop is triggered to the subtract mode; also, if required, a preshift pulse is applied to both the $M$ and $L$ registers and the $S M$ flip-flop is turned off. Under normal conditions, the 12 shift pulses $\mathrm{S}$ $\left(S_{1}-S_{12}\right)$ are used to carry out the arithmetic operation (addition or subtraction) between the $\mathrm{C}$ register and the $M$ register. It should be noted, however, that, if the three bits of the $L$ register are equal, the above operation does not take place, as the pulse counter has been advanced 12 places with Pulse B. Pulse $\mathrm{X}$ is now used to produce the first right shift of the $M$ and $L$ registers, if a preshift pulse has not been applied (SM flip-flop still on). Finally, the Y pulse

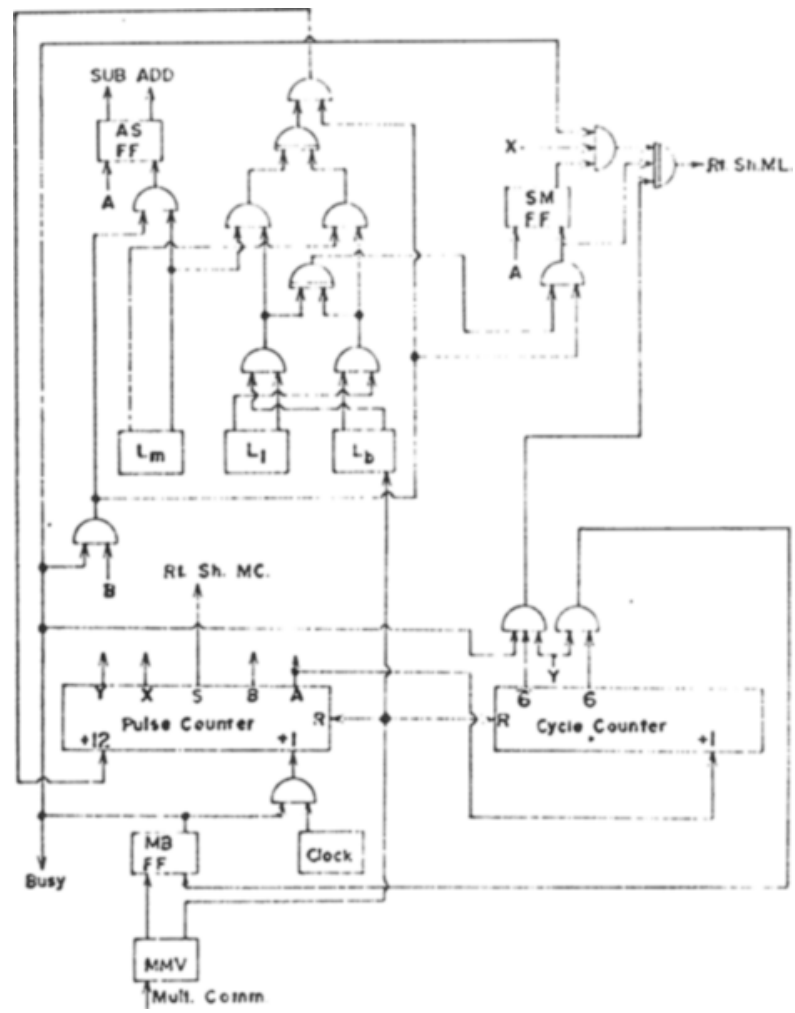

Fig. 2. Multiplier controller.

is used to produce the necessary second right-shift pulse. All of the first five cycles are identical to the above. However, the sixth cycle is slightly different, in that the last right-shift pulse is inhibited by the cycle counter having reached the count of six; in this case, the $Y$ pulse is used to reset the MB flip-flop, stopping the pulse counter and signaling that the operation is complete.

\section{DIVISION}

In the case of division, the process is more complex and, probably due to its relatively low frequency of use, less attention appears to have been taken in its development. Therefore, dividers tend to be serial in mode, with the arithmetic being carried out in the same form as that used in the multiplier. Consequently, the method to be used is that given by Booth and Booth (1965), which is a nonrestoring method giving a correctly signed quotient for 2's complement signed numbers for divisor and dividend. The total time for a 12-bit word will be

Table 2

Division RuJes

\begin{tabular}{ll} 
& Division Rules \\
\hline Sign Bits & \multicolumn{1}{c}{ Arithmetic Operation } \\
\hline Equal & $\begin{array}{l}\text { Subtract and add } 1 \text { to the } \\
\text { quotient. }\end{array}$ \\
Unequal & \begin{tabular}{l} 
Addition only. \\
\hline
\end{tabular} \\
\hline
\end{tabular}

approximately 176 microsec, using a clock rate of $1 \mathrm{MHz}$. In this method the sign bits of the divisor and dividend are compared, and the action to be carried out is given in Table 2 , in which the arithmetic operation is between the divisor and dividend; however, this operation must not take place until after there has been a left shift of the dividend and quotient registers. In the final cycle it is only necessary to complement the sign bit of the quotient, and some rounding-off procedure may be included; in this particular case, no rounding-off procedure has been provided, as the remainder is available if required.

The logic diagram of the algorithm controller is given in Fig. 3. Initially, the divisor is loaded into the $\mathrm{C}$ register, the dividend into the $M$ register, and the $\mathrm{L}$ register is cleared. At the same time as the dividend is loaded, the two counters are reset to zero, the AS flip-flop to the add mode, and the divider operation initiated similarly to that of the multiplier. A typical cycle is as follows: Pulse A advances the cycle count by unity and tests the sign bits of the $C$ and $M$ registers to determine the arithmetic operation to be performed. If the sign bits are equal, the AS flip-flop is set to the subtract mode and a 1 is inserted into the least significant bit $\left(\mathrm{L}_{Q}\right)$ of the $L$ register. At Pulse Time $B$, a single 


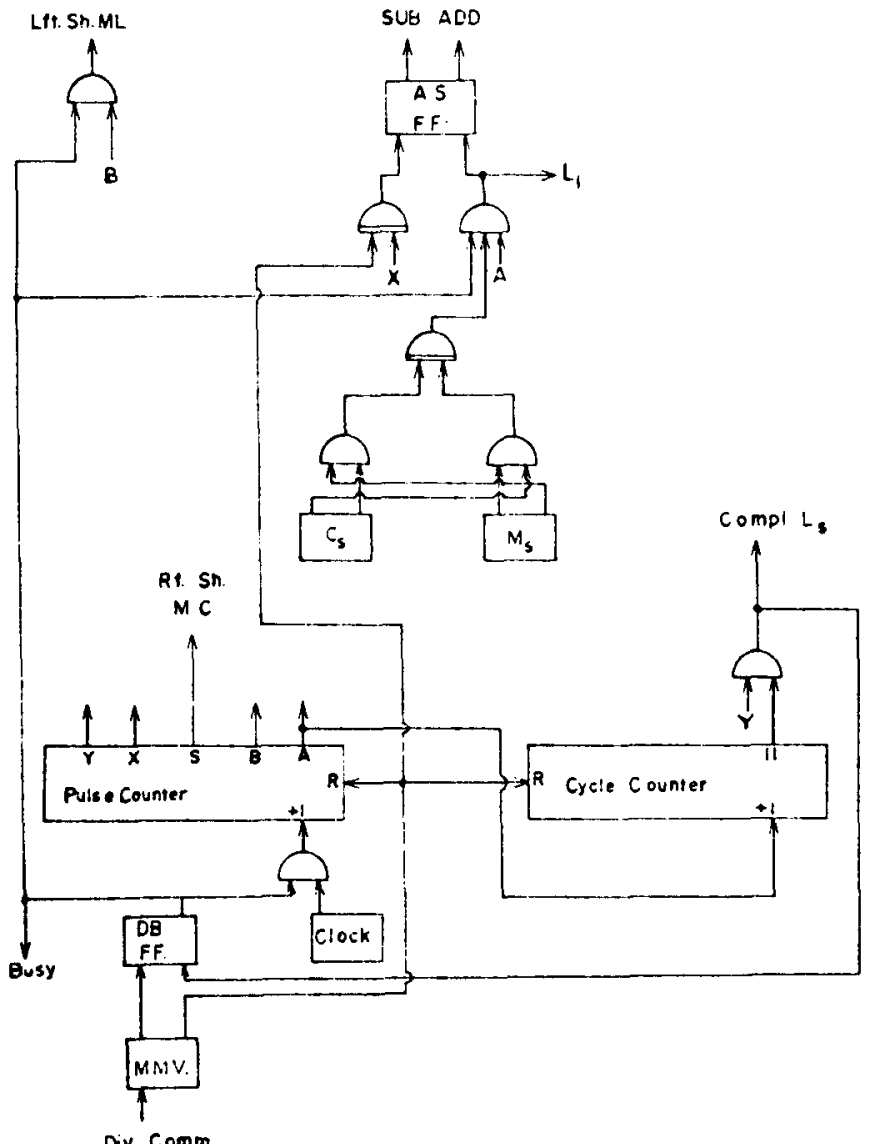

Fig. 3. Divider controller. left shift of the $M$ and $L$ registers takes place, followed by the appropriate arithmetic operation between the $\mathrm{C}$ and $M$ registers, using the 12 shift pulses (S). Pulse $X$ is then used to reset the AS flip-flop to the add mode for the next cycle. In the case of the final cycle (11th), the sign bit $\left(L_{s}\right)$ of the quotient is complemented by the $\mathrm{Y}$ pulse and the DB flip-flop reset, stopping the pulse counter and signaling that the operation is complete.

FINAL CONSIDERATIONS

Although the final design is still incomplete, the estimated material cost using $\mathrm{R}$-series modules is about $\$ 1,500$, including the two device selectors for loading and unloading the data. If the divider were not included, the cost would be reduced to about $\$ 1,200$, the main difference being due to the left-shift requirement for the $M$ and $\mathrm{L}$ registers if division is to be performed. If B-series modules were used, the speed of operation should be increased tenfold at about twice the cost. However, it should be borne in mind that the total time to load the data and extract the result would be about 150-200 microsec, so that reducing the multiplier or divider times is a questionable expense.

The two busy flip-flops are necessary to the operation of the unit, and, therefore, sensing of the appropriate flip-flop may be carried out by the programmer if he wishes to do so before extracting the result of the operation being performed.

Finally, it should be pointed out that, though designed primarily for the PDP $-8 / S$, the unit may be equally used on any member of the PDP-8 family of computers, it being understood that it is slower, but also cheaper, than the EAE.

The authors believe that this unit will be a useful addition to the PDP-8/S in particular and to the PDP-8 family in general.

\section{REFERENCES}

BOOTH, A. D. \& BOOTH, K. H. V Automatic digital calculators. (3rd ed.) London: Butterworth, 1965.

THOMAS, P. A. V. The design philosophy of a small electronic automatic digital computer. PhD thesis, University of Glasgow, Scotland, 1961. 